\title{
PSD performance and charge reconstruction with DAMPE
}

\author{
Yapeng Zhang*1 ${ }^{*}$ Yongjie Zhang ${ }^{1,2}$, Tiekuang Dong ${ }^{3}$, Pengxiong Ma $^{2,3}$, Yuhong $\mathbf{Y u}^{1}$, \\ Paolo Bernardini ${ }^{4,5}$, for the DAMPE collaboration \\ ${ }^{1}$ Institute of Modern Physics, Chinese Academy of Sciences, Lanzhou 730000, China \\ ${ }^{2}$ University of Chinese Academy of Sciences, Beijing 100049, China \\ ${ }^{3}$ Key Laboratory of Dark Matter and Space Astronomy, Purple Mountain Observatory, \\ Chinese Academy of Sciences, Nanjing 210008, China \\ ${ }^{4}$ Università del Salento - Dipartimento di Matematica e Fisica "E. De Giorgi", I-73100, \\ Lecce,Italy \\ ${ }^{5}$ Istituto Nazionale di Fisica Nucleare (INFN) - Sezione di Lecce, I-73100, Lecce, Italy \\ E-mail: $\mathrm{y \cdot P.zhangdimpcas.ac.cn}$
}

\begin{abstract}
The DArk Matter Particle Explorer (DAMPE) is a satellite-borne device for detecting high energy electrons, gamma-rays, protons and heavy-ions in space. The Plastic Scintillator Detector (PSD) is the top-most of the four sub-detectors of DAMPE, which was designed to measure the charge of incident high-energy particles. It also serves as a veto detector for discriminating gamma-rays from charged particles. In this paper, we first introduce the structure and on-orbit operation status of the PSD. The calibration procedures, including the calibration of the pedestal, the dynode ratio, the detector alignment and the energy reconstruction, are then presented. Based on on-orbit data, the preliminary charge spectrum is obtained.
\end{abstract}

35th International Cosmic Ray Conference - ICRC2017

10-20 July, 2017

Bexco, Busan, Korea

\footnotetext{
* Speaker.

$\dagger$ The DAMPE mission is funded by the strategic priority science and technology projects in space science of Chinese Academy of Sciences. In China, the data analysis is supported in part by the National Key Research and Development Program of China (No. 2016YFA0400200 and 2016YFA0400201), National Basic Research Program of China (No. 2013CB837000), National Natural Science Foundation of China (No. 11673047, 11673075 and 11303107). In Europe, the data analysis is supported by the National Institute for Nuclear Physics (INFN), Italy.
} 


\section{Introduction}

Measuring the composition of cosmic-ray nuclei is important for studying their origin, as well as the propagation of the cosmic-ray in the interstellar medium [四]. The DArk Matter Particle Explorer (DAMPE) [[]], launched on December 17th, 2015, is a satellite-borne calorimeter type particle detector for detection of electrons, $\gamma$-rays, and cosmic-ray nuclei. The DAMPE detector consists of four sub-detectors, a Plastic Scintillator Detector (PSD), a Silicon-Tungsten tracKerconverter (STK) [B]], a BGO imaging calorimeter [四], and a NeUtron Detector (NUD). The combination of these four sub-detectors allows for a good measurement of the charge, direction and energy of an incident particle. It also provides an efficient lepton/hadron separation. The charge measurement is done independently by the PSD and the STK.

In this work we present the structure, operation, calibration and charge reconstruction of the PSD instrument. The charge measurement with the STK can be found elsewhere [\$]].

\section{Plastic scintillator detector}

The PSD is the top-most sub-detector of the DAMPE, which is designed to fulfill two major tasks: (a) to measure the charge of incident high-energy particles with the charge number $Z$ from 1 to 26 and more; (b) to serve as a veto detector for discriminating $\gamma$-rays from charged particles. All these require the PSD to have a large dynamic range, good energy resolution and high detection efficiency.

The PSD is made by two layers of plastic scintillation array detector, as shown in Fig. $\mathbb{\text { m. Each }}$ layer is composed of 41 detection modules, which include a plastic scintillator (EJ200) bar and one photomultiplier tube (PMT) at each end. The dimension of bars is $884 \mathrm{~mm} \times 28 \mathrm{~mm}(25$ $\mathrm{mm}$ for bars at edges) $\times 10 \mathrm{~mm}$. In order to avoid the ineffective detection area of each layer, the neighboring bars in each layer are staggered by $8 \mathrm{~mm}$, as shown in Fig. ㅁ. The active detection area of the PSD is $825 \mathrm{~mm} \times 825 \mathrm{~mm}$. The bars in the top and bottom layers are parallel to the $X$-axis and the $Y$-axis of the DAMPE coordinate system, respectively. For covering an energy dynamic range from 0.1 MIPs to 1400 MIPs, signals from the dynode 5 (Dy5) and the dynode 8 (Dy8) of each PMT were extracted. In order to obtain a stable on-orbit performance of the PSD, an active temperature control strategy was implemented by using front-end electronic boards and additional heat coils as thermal sources. More detailed information about design, assembly and lab tests can be found in Refs. [6, 四].

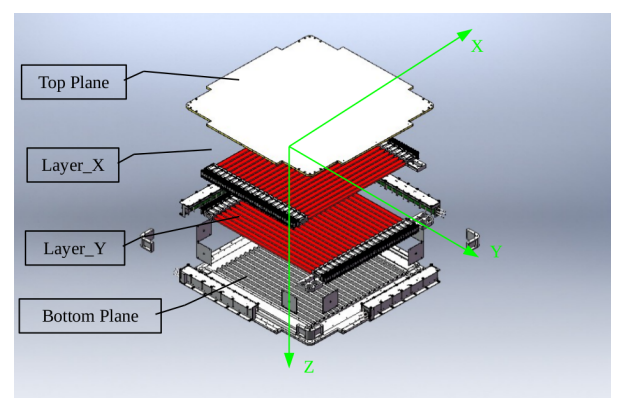

Figure 1: The structure of the PSD. 


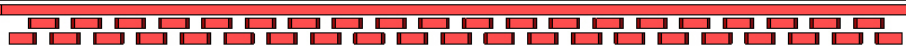

Figure 2: Side view of the PSD.

\section{On-orbit status of the PSD}

As the top-most sub-detector of the DAMPE, the temperature of the PSD is sensitive to the sun light change. Fig. [1] shows the temperature (vertical axis) at $+X$-direction of the PSD versus the date (horizontal axis). The lowest temperature at the end of February 2016 is because that the the effective area of the DAMPE for receiving the sunlight is the minimum. Later, the temperature increased with the increasing effective area. Until about April 25th, 2016, the satellite has partially been in the shadow of the Earth in each orbit, and the temperature is stabilized due to the balance of these two effects. Two solid horizontal lines in the figure indicate a range of $1^{\circ} \mathrm{C}$. Such small temperature variation shows that the temperature control system works well.

In order to characterize the $H V$ stability of the PSD in space, a HV variation quantity $(H V-$ Mean)/Mean is defined, where $H V$ is the temperature of a side of the PSD of a given day, the Mean is the mean value of the HV of all times (days). Fig. 目 shows the HV variation (vertical axis) versus time. The HV variations of PMT 22-40 at positive side of top layer (X-layer) are presented. For a display reason, offsets are added to the correlations. In this plot, HVs of 8 PMTs fluctuate with the same pace (near March, 2016), because these PMTs are powered by the same HV channel. In general, the rate of $\mathrm{HV}$ change is less than $0.5 \%$, which brings a solid base for a stable performance of the PSD in space.

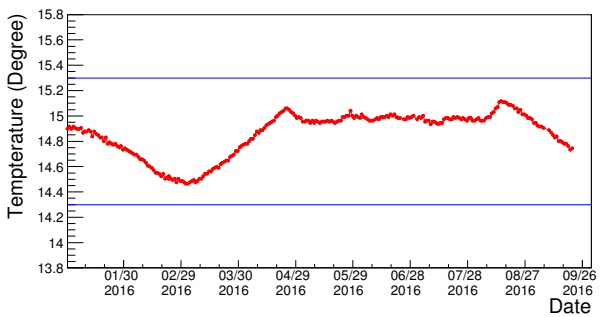

Figure 3: The Temperature of one side of the PSD versus the date. Two solid horizontal lines indicate a range of $1^{\circ} \mathrm{C}$.

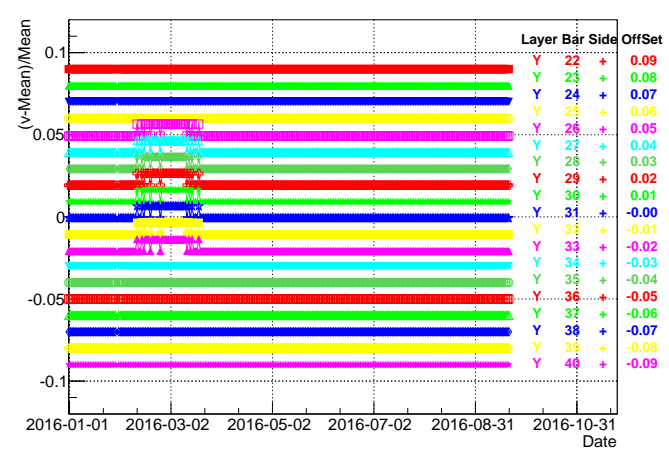

Figure 4: HV change rate (vertical axis) versus the date.

\section{Calibration of the PSD}

The purpose of the PSD calibration is to convert the recorded ADC values to the energy depositions induced by incoming charged particles, in which the noise and the non-linearity in actual detection processes are taken into account. The PSD calibration includes several aspects: the pedestal 
calibration, calibrating the dynode ratios of PMTs, obtaining the attenuation function and reconstructing the energy deposition. During the calibration procedure, the data collected in the South Atlantic Anomaly (SAA) region are excluded. Calibration steps mentioned above are discussed in the following sub-sections.

\subsection{Pedestal calibration}

The pedestal is the baseline of the real signal, which needs to be removed before the data analysis. The pedestals of the PSD are sampled twice per orbit with random triggers. For each PSD readout channel, its pedestal distribution can be well fitted with a Gaussian function. Fig. 1 shows typical pedestal distributions of dynode 5 and dynode 8 of a PMT. The fitted Gaussian functions are depicted by the red solid curves, respectively. For each channel, the mean of the pedestal is daily updated, and it is subtracted from the non-pedestal mode data. Fig. 6 shows the pedestal variation (see HV variation) versus date. In general, the pedestals of the PSD are quite stable and the overall variation is less than $0.1 \%$ during the first running year.

The mean value of PSD pedestal are investigated before and after the launch of the DAMPE. Fig. $\square$ shows pedestal values (vertical axis) for all readout channels of the positive side of the top layer of the PSD. The circles represent the values measured on Dec. 15, 2015 before the launch and the triangles are the values measured on Dec. 20, 2015 after launch. This shows that the PSD pedestal values are almost the same before and after the launch.
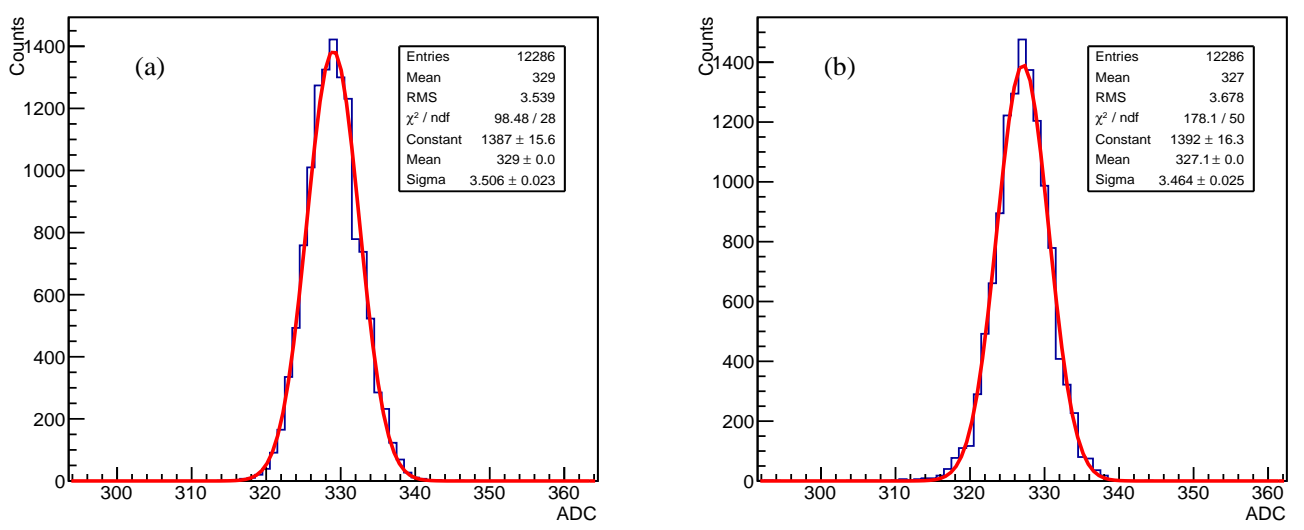

Figure 5: A typical pedestal distributions of dynode 5 (a) and dynode 8 (b) of a PMT, the fitted Gaussian functions are depicted by the red solid curves.

\subsection{Dynode ratio calibration}

As mentioned in section 凤, in order to have a large dynamic range of the energy measurement, the signals from dynode-5 (Dy5) and dynode-8 (Dy8) of each PMT of PSD are extracted. After subtracting the pedestals, the ADCs of Dy5 and Dy8 of the same PMT show a good linear

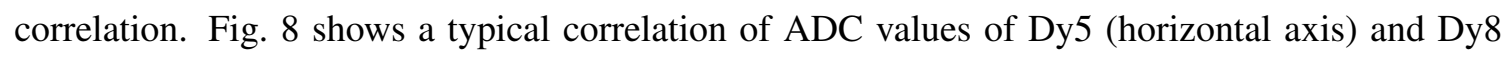
(vertical axis) of a PMT, which is fitted by a linear function. By using the obtained slope parameter $k$ (dynode ratio) and intersection parameters of each PMT, the two measurements of Dy5 and Dy8 


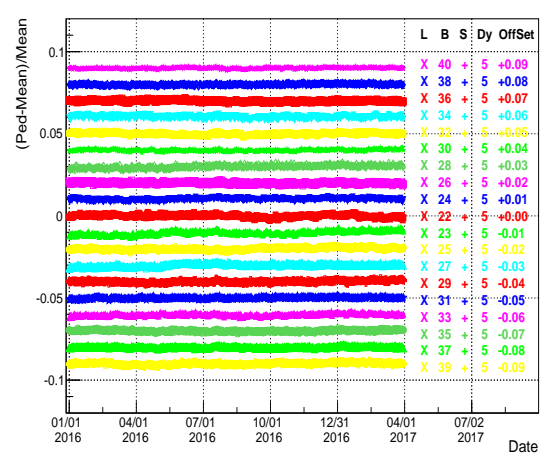

Figure 6: Pedestal variation versus date.

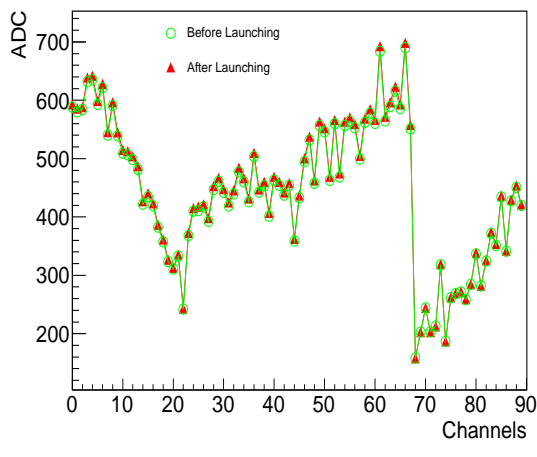

Figure 7: Pedestals before (green circles) and after (red triangles) the launching of DAMPE.

are processed into a single value, which is expressed as following

$$
A D C=\left\{\begin{array}{ll}
A D C_{8} & \left(A D C_{8} \leq 11000\right) \\
k \times A D C_{5}+C & \left(A D C_{5}>11000\right)
\end{array},\right.
$$

where $A D C_{5}$ and $A D C_{8}$ are $\mathrm{ADC}$ values of Dy5 and Dy8 of a PMT of the PSD, respectively.

\subsection{Light attenuation calibration}

According to the Bethe-Bloch formula, the energy deposition of proton MIPs in the PSD can be used as a unit to measure the energy depositions of high energy cosmic-ray particles at first order. Since the scintillation light generated in PSD bars is attenuated during its propagation, the detected responses of MIPs depend on the hit positions.

In order to investigate the attenuation effect of PSD bars, the MIP events are selected firstly. An event is classified to be a MIP event if it can pass through all 14 BGO layers and the energy depositions corrected for the incidence angle in each BGO layer are around the energy deposition range of MIPs ( $1 \mathrm{MIP}=23 \mathrm{MeV}$ for BGO bar with a thickness of $25 \mathrm{~mm}$ ). For MIP events with only one STK track, the hit position are determined by back-tracing the track. The light attenuation behavior of each PSD bar is investigated by correlating the obtained hit position along the strip and its ADC values. Fig. $Q$ shows a typical scattering plot of hit position (horizontal axis) and path-length corrected ADC value (vertical axis) for one side of a PSD bar. The ADC distribution in each position bin still obeys the Landau distribution. The MPV values are obtained by fitting the ADC distributions in each X-bin with a Gaussian convoluted Landau function, which are plotted by red triangles in Fig.Q. The attenuation function for each side of PSD bars is obtained by fitting the corresponding distribution of MPV value with the function

$$
A(x)=C_{0} e^{-x / \lambda}+C_{1}+C_{2} x+C_{3} x^{2}+C_{4} x^{3},
$$

where $x$ is the hit position along a PSD bar, $C_{0}-C_{4}$ and $\lambda$ are free parameters. The attenuation functions are used in the energy reconstruction. 

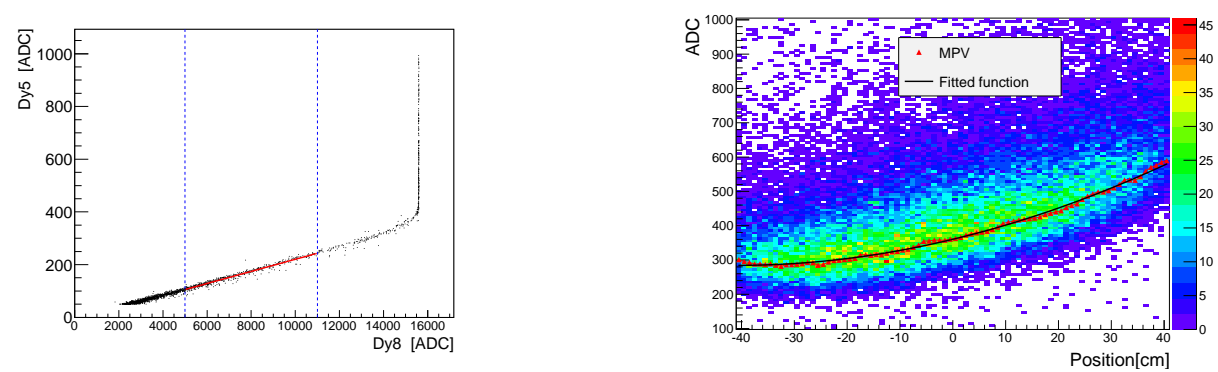

Figure 8: The correlation of pedestal subtracted Figure 9: ADC value (vertical axis) of MIPs verADC values of Dy5 (vertical axis) and Dy8 (hor- sus the hit position (horizontal axis). The red triizontal axis) of a PSD PMT, the red line is the angles are the MPV values of ADC distributions fitted linear function. of position bins, the black solid line is the fitted attenuation function.

\section{PSD Alignment}

Detector alignment is important for improving the charge resolution and the gamma ray event identification capability of the PSD. For charge reconstruction, the proper PSD alignment can improve the accuracy of the reconstructed energy. For gamma ray event identification, the alignment can improve the match probability of the track and the right PSD bar.

For each PSD bar, we assume that there is a 3-dimensional shift $\delta$ and a rotation angle $\theta$. The energy loss of the incident charge particle is in proportion to its path length inside the volume of a bar. With varying $\delta$ and $\theta$, one can obtained the passing length of a given track, and then the measured energy deposition can be corrected. By comparing the energy spectrum in $1 \mathrm{~mm}$ of vertical injected events and the one of passing-corner events iteratively, one can obtained the best values of the shift parameters $\delta$ and the rotation $\theta$ for each PSD bar. Fig. 10 shows the energy spectrum (blue line) of passing-corner events before PSD alignment and the one (red line) after the alignment.

\section{Energy and charge reconstruction}

Considering the typical energy loss of proton MIPs is about $2 \mathrm{MeV} / \mathrm{cm}$ (the thickness of the PSD bar is $1 \mathrm{~cm}$ ), the energy deposition at left/right side of a PSD bar $E_{L / R}$ is derived as following

$$
E_{L / R}=\frac{A D C_{L / R}}{A(x)_{L / R}} \times 2 \mathrm{MeV},
$$

where $A D C_{L / R}$ is the path length corrected ADC value, $A(x)_{L / R}$ is the corresponding attenuation function (see Eq. 4.2) and the hit position $x$ is given by the track. The charge of incident particles could be derived from the reconstructed energy, described as following,

$$
\begin{aligned}
Q_{L / R}^{r e c} & =\sqrt{\frac{E_{L / R}}{E_{M I P}}}, \\
Q_{L / R} & =f\left(Q_{L / R}^{r e c}\right),
\end{aligned}
$$


where $Q_{L / R}^{r e c}$ is the reconstructed charge of incident particle on both ends of a PSD bar, $E_{L / R}$ is the reconstructed energy, $E_{M I P}=2 \mathrm{MeV}$ is the defined energy deposition of MIPs per cm, $Q_{L / R}$ is the corrected charge and $f(Q)$ is the quenching effect correction function. The quenching behavior of a PSD bar for heavy nuclei is described by Birks' semi-empirical formula [ [] ], $f(Q)$ is a parameterized Birk's law fitted from observed charges and the nominal charges of cosmic-ray elements. Fig. 1 shows a charge spectrum reconstructed by the PSD using one year flight data, the charge peaks of major elements are clearly visible (the last peak is ${ }^{28} \mathrm{Ni}$ ).

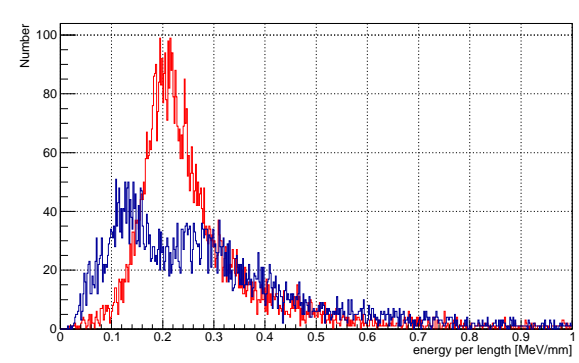

Figure 10: Energy spectrum of passing-corner events Figure 11: The charge spectrum reconstructed by the before (blue) and after (red) the PSD alignment.

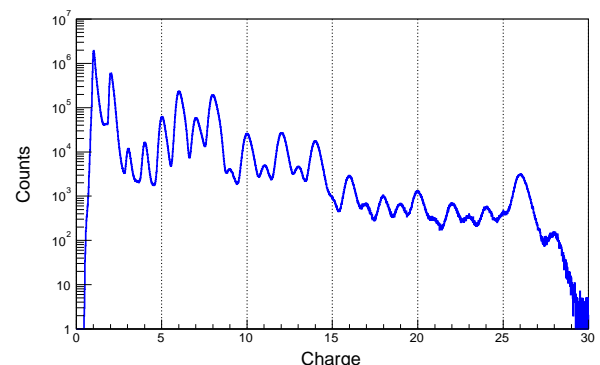

top layer of the PSD.

\section{PSD detection efficiency}

Electromagnetic shower can be well separated from the hadronic shower by the BGO calorimeter. The flux of the cosmic $\gamma$-ray is about 1000 times lower than the one of the electron, therefore the detection efficiency of the PSD is crucial for discriminating $\gamma$-rays from electrons. The PSD detection efficiency is evaluated using MIP events which is required to have only one good STK track. Good STK track means that the track is the only track in the event and it has at least 5 valid clusters in both $X-Z$ plane and $Y-Z$ plane. The matched PSD bars can be found by extrapolating the track to each sub-layer of the PSD. The detection efficiency $\eta_{l, b}$ of $b$-bar in $l$-layer is defined as following,

$$
\eta_{l, b}=N_{l, b}^{\text {Fired }} / N_{l, b}^{\text {STK }},
$$

where $N_{l, b}^{S T K}$ indicates how many times the bar matches with the STK track, $N_{l, b}^{\text {Fired }}$ indicates how many times that bar is fired. The firing condition for the PSD bar is $E_{b a r}>0.5 \mathrm{MeV}$. Fig. 12 shows the detection efficiency (vertical-axis) of the bars in the bottom PSD-layer. The detection efficiency of a single PSD bar is more than $99.1 \%$.

\section{Summary}

The DAMPE has been operated in space for over one year, the modules of the PSD in space are working as expected. The temperature variation of the PSD is less than $1^{\circ} \mathrm{C}$ and the $\mathrm{HV}$ variation for each PMT is less than 0.5\%. The active temperature-control system used to minimize the temperature variation of the PSD has proven to be effective.

The calibration procedure of the PSD and the method used to reconstruct the charge of the incident particles are described. The detection efficiency has been investigated and it results higher 


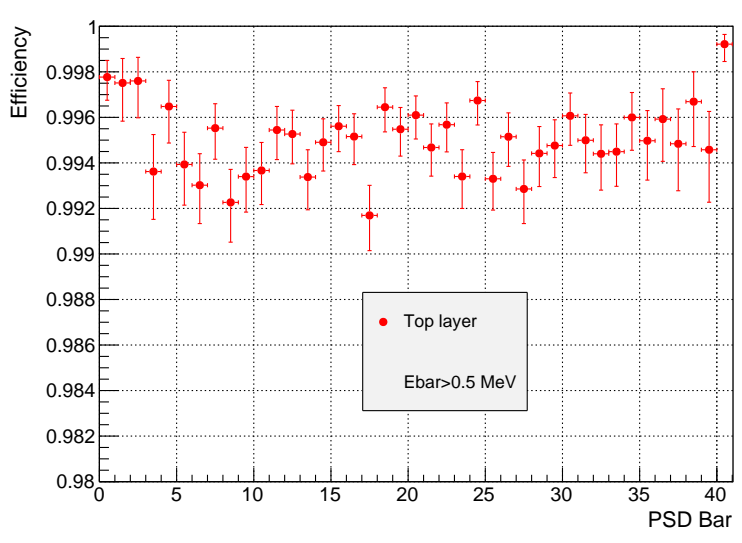

Figure 12: The detection efficiency of the PSD.

than $99.1 \%$ for all bars. A preliminary charge spectrum measured by the top layer of the PSD is shown, the cosmic nuclei from $\mathrm{H}$ to $\mathrm{Ni}$ are clearly visible. With such a high charge resolution we can select pure samples of each element to do further analysis.

\section{References}

[1] Blasi, P., Astron Astrophys Rev 21 (2013): 70.

[2] J. Chang et al. [DAMPE Collaboration], The Dark Matter Particle Explorer mission, Astropart. Phys. submitted (2017). [arXiv:1706.08453].

[3] P. Azzarello, et al. Nuclear Instruments and Methods in Physics Research Section A: Accelerators, Spectrometers, Detectors and Associated Equipment 831 (2016): 378.

[4] Feng, Changqing, et al. IEEE Transactions on Nuclear Science 62.6 (2015): 3117-3125.

[5] S.Vitillo et al, Measurement of cosmic rays charge with DAMPE Silicon-Tungsten Tracker, Poster at ICRC2017.

[6] Yuhong Yu, et al. arXiv:1703.00098v1 [astro-ph.IM].

[7] Zhou, Yong, et al. Nuclear Instruments and Methods in Physics Research Section A: Accelerators, Spectrometers, Detectors and Associated Equipment 827 (2016): 79-84.

[8] J.B. Birks, Theory and Practice of Scintillation Counting, Pergamon Press, London, 1964. 